\title{
Experience with the use of handheld Doppler for fetal heart rate monitoring during coronavirus disease-19 pandemic
}

\author{
Devina Bajpayee 1 , Ritu Agrawal', Sourav Ghosh Dastidar ${ }^{3}$, Nitin Bisht ${ }^{4}$, Ranjan Kumar Panda \\ Arvind Kumar ${ }^{6}$, Enisha Sarin ${ }^{7}$, Harish Kumar ${ }^{8}$ \\ From ${ }^{1}$ Senior Advisor-Maternal Health, ${ }^{2}$ Senior Technical Advisor-Obstetrics and Gynaecology, ${ }^{3}$ State RMNCH+A Advisor, ${ }^{4}$ State Technical Advisor, \\ ${ }^{5}$ State Technical Advisor, ${ }^{6}$ Senior Data Manager, ${ }^{7}$ Senior Advisor-M\&E, ${ }^{8}$ Project Director, Department of Health, Nutrition and WASH, IPE Global, \\ New Delhi, India
}

Correspondence to: Enisha Sarin, B-84, Defence Colony, New Delhi - 110 024, India. E-mail: esarin@ipegllobal.com

Received - 06 October 2020

Initial Review - 10 October 2020

Accepted - 12 October 2020

\section{ABSTRACT}

Background: Fetal heart rate (FHR) monitoring during intrapartum care is important; however, the use of Doppler during coronavirus disease (COVID-19) pandemic needs to be investigated especially in light of the enhanced infection prevention (IP) required during labor and childbirth. The present study was carried out to evaluate the experience of using a handheld Doppler having both continuous and intermittent monitoring features in seven health facilities across three states. Materials and Methods:Program data including case load, frequency of monitoring, and fetal outcome was obtained from January to March 2020 were compared with data during the lockdown period from April to June 2020. A semi-structured questionnaire was used for a telephonic qualitative interview of eight service providers from the seven facilities to supplement the quantitative data. Transcripts were analyzed using ATLAS.ti to find out how the Doppler was being used. Results: There was decrease in the case load during lockdown; however, there was no decrease in the frequency of monitoring. There was no difference on rate of abnormal FHR detected, stillbirths, and asphyxia. IP protocol was followed in all facilities. The handheld Doppler was used by four facilities to continuously monitor pregnant women. While, study participants reported that strapping the belt for continuous monitoring reduced exposure to suspected COVID cases, two participants believed that strapping could be a risk factor for transmission. Conclusion: Usage of the Doppler for FHR detection can be used for monitoring progress during labor using IP protocol.

Key words: Coronavirus disease-19, Doppler, Fetal heart rate monitoring, India, Maternal health, Newborn health

$\mathrm{C}$ hildbirth is a normal physiological phenomenon; however, in developing countries during intrapartum period, there is an increased risk of mortality for the mother and the newborn due to preventable causes. Over one-third of maternal deaths and life-threatening conditions [1,2], approximately half of all stillbirths and a quarter of neonatal deaths result from complications during labor and childbirth [3]. In India, 32,000 maternal and 640,000 newborn deaths occur during childbirth every year [4,5]. Prolonged intrapartum fetal hypoxia during labor often results in fresh stillbirth or a severely asphyxiated neonate. Therefore, intrapartum fetal heart rate (FHR) monitoring is crucial for the early detection of fetal hypoxia. However, FHR is inadequately monitored across developing countries [6,7].

In addition, there is an increased risk of coronavirus disease (COVID-19) infection with complications among pregnant women who have chronic diseases or maternal complications as they are susceptible to respiratory pathogens [8]. For those with the virus, there is an increased risk of preterm rupture of membranes, preterm delivery, and intrapartum FHR abnormalities [9-13]. Childbirth during this period is fraught with danger for both the pregnant woman as well as health-care providers. Active labor requires a woman to exert effort increasing the possibility of aerosol generation which puts the health-care team at risk. It is recommended that labor and delivery personnel should be granted the protection they need for themselves and other patients [14]. Close contact being the primary driver of COVID-19 infection, limiting frequency, and duration of exposure in labor units is instrumental in prevention for health-care personnel [15].

To improve FHR monitoring, the USAID Vriddhi project introduced an FDA approved handheld Doppler in seven facilities of three aspirational districts across Jharkhand, Odisha, and Uttarakhand. A baseline assessment of the same facilities revealed that only a third of cases were monitored during first stage of labor at frequencies recommended by the WHO. None of the cases were monitored as per standard guidance of care during the second stage. High case load and the time required for monitoring were reported as challenges in adhering to standard monitoring protocols. While Doppler's were used in conjunction with stethoscope, limitations in readings due to fluctuations were reported in the devices being used.

The FHR monitoring device (Laerdal made) uses a nine crystal Doppler ultrasound sensor to measure and analyze the FHR. Faster, 
easier, and reliable monitoring improves the quality of monitoring practices. The sensors give the device an increased range to quickly detect FHR in a few seconds over a large area. The inbuilt system displays the FHR on a monitor, using a traffic signal system and provides an alarm in the case of prolonged abnormal FHR. The device was introduced in a total of seven facilities -3 Community Health Centre (CHC), three district hospital, and one medical college in August 2019. During implementation, routine facility level data and data on related indicators were regularly collected every month. Timely monitoring and supervision were provided by Vriddhi project. Improvement in FHR monitoring has been seen in program data. Quarterly provider feedback also reveals satisfaction in using the Doppler. Furthermore, interviews with beneficiary brought out an improved experience that they were able to squat and sit and be mobile even when device was strapped on for prolonged monitoring.

In March 2020, due to COVID-19 Pandemic, with the nationwide shutdown, there was a strong possibility that the routine maternal and newborn health-care services would be impacted. Field level hand holding and supervision support were withheld. However, the project continued their support remotely using telephone and other modes of digital communication. In addition, remote facilitation of training on infection prevention (IP) and the cleaning and disinfection of device and its accessories between every use was reiterated. The experience of care delivery during this period among service providers was not known. Issues about how the intervention Doppler was used during this period and whether FHR monitoring was affected became important questions. In this regard, a quick assessment was conducted with objectives to compare the frequency of FHR monitoring and fetal outcomes between pre and during COVID pandemic and to understand the experience of service providers in monitoring practices during COVID times.

\section{MATERIALS AND METHODS}

Program data from January to June 2020 of the state approved USAID Vriddhi project that routinely gets transferred to the project national office were assessed retrospectively. The data were divided into pre COVID (January-March 2020) and COVID lockdown period (April-June 2020). Frequency of monitoring was assessed through sample case sheets. Indicators such as total delivery, total C-section, total abnormal FHR detected, and frequency of monitoring were compared between two groups.

The qualitative aspect was conducted with the help of an interview guide. Only those participants who used the Doppler for monitoring with a willingness to participate in the study were included in the study. A total of eight participants mainly staff nurses and resident medical doctors were selected from all the facilities from three states and an informed consent was obtained before the interview. An interview of 15-20 min duration was conducted remotely over telephone by a senior technical staff of Vriddhi, from last week of May to the $1^{\text {st }}$ week of June and was recorded with permission of participants. The objective of the study was explained at the outset of the interview. Questions were asked in relation to the prescribed format to understand the feasibility, ease of use and
IP practices in COVID times. In addition, to validate the technical use of the handheld Doppler, a few specific questions were asked to understand any challenges in its use. Technical suggestions were given by the interviewer at the end of each interview. Qualitative data were analyzed on ATLAS.ti.

\section{RESULTS}

Data on total delivery, total C-section, outcomes of delivery, and FHR monitoring frequency for previous period and during lockdown are provided in Tables 1 and 2. However, during the period of lockdown, sample case sheets in the facilities in Odisha could not be reviewed. Thus, the results relating to frequency of monitoring are not provided for two out of the seven facilities. During the study period, there was slight decrease in the frequency of monitoring and number of cases never monitored during COVID lockdown as compared to pre-COVID time. There was $20.6 \%$ decrease in the total number of deliveries during COVID lockdown as compared to the previous quarter. There was no significant difference in the number or proportion of c sections conducted, stillbirth, asphyxia, and rate of abnormal FHR detected between two periods.

Data for the Regional Institute of Medical Science (RIMS) are shown in Table 3 as it was the only facility that had COVID positive pregnant women coming for delivery during that period of time. Due to beginning of surge of COVID in India and travel restrictions in March $23^{\text {rd }}$, there was decrease in the total delivery cases (326) in march compared to the previous monthly average of 600 cases (September 2019-February 2020). As seen in the tables above, the frequency of monitoring at least three times or more has only slightly decreased during COVID lockdown. During the same time, cases never monitored also reduced. The total number of deliveries is clearly less than the previous quarter- percent decrease is $20.6 \%$.

Table 1: FHR monitoring indicators pre and during COVID lockdown

\begin{tabular}{lcc}
\hline Indicators & $\begin{array}{c}\text { January-March } \\
\mathbf{2 0 2 0} \text { (n and \% of } \\
\text { total sample cases) }\end{array}$ & $\begin{array}{c}\text { April-June 2020 (n } \\
\text { and \% of total sample } \\
\text { cases) * }\end{array}$ \\
\hline $\begin{array}{l}\text { Sample cases taken } \\
\text { for checking FHR } \\
\text { monitoring }\end{array}$ & 2794 & 2136 \\
Monitored $\geq 3$ times & $1048(37.5 \%)$ & $698(32.7 \%)$ \\
Never monitored & $134(4.8 \%)$ & $17(0.8 \%)$ \\
\hline *excluding two facilities. FHR: Fetal heart rate &
\end{tabular}

Table 2: Delivery related indicators pre and during COVID lockdown

\begin{tabular}{lcc}
\hline Indicators & $\begin{array}{c}\text { January-March } \\
\mathbf{2 0 2 0}(\mathbf{n} \text { and \% of } \\
\text { total delivery) }\end{array}$ & $\begin{array}{c}\text { April-June 2020 } \\
\text { (n and \% of total } \\
\text { delivery) }\end{array}$ \\
\hline Total delivery & 5755 & 4566 \\
Emergency C-section & $1241(21.6 \%)$ & $903(19.8 \%)$ \\
Abnormal FHR detected & $198(3.4 \%)$ & $161(3.5 \%)$ \\
Stillbirths & $73(1.3 \%)$ & $66(1.4 \%)$ \\
Asphyxia & $437(7.6 \%)$ & $295(6.5 \%)$ \\
\hline
\end{tabular}

FHR: Fetal heart rate 
Table 3: Delivery cases, outcomes, and frequency of monitoring at RIMS, Medical College, Ranchi

\begin{tabular}{lcc}
\hline Indicators & $\begin{array}{c}\text { January-March } \\
\mathbf{2 0 2 0}\end{array}$ & $\begin{array}{c}\text { April-June } \\
\mathbf{2 0 2 0}\end{array}$ \\
\hline Total delivery & 1503 & 878 \\
Emergency C-section & $808(53.8 \%)$ & $395(44.9 \%)$ \\
Abnormal FHR detected & $50(3.3 \%)$ & $49(12.4 \%)$ \\
Stillbirth & $26(1.7 \%)$ & $8(0.9 \%)$ \\
Asphyxia & $194(12.9 \%)$ & $119(13.5 \%)$ \\
Sample cases taken for checking & 360 & 288 \\
FHR monitoring & & \\
Monitored $\geq 3$ times & $192(53.3 \%)$ & $155(53.8 \%)$ \\
Never monitored & $8(2.2 \%)$ & $0(0.0 \%)$ \\
\hline FHR: Fetal heart rate & &
\end{tabular}

FHR: Fetal heart rate

However, on the other measures, the difference is not significant. The difference in the number of $\mathrm{C}$ - sections conducted, stillbirth, asphyxia and rate of abnormal FHR detected is not vastly different in the two periods. Significant decrease (42\%) in the number of delivery was observed during the lockdown period. Lowest number of cases was recorded in May (182 cases from previous 433), thereafter the total delivery increased to 263 in June. Similarly, percent $\mathrm{C}$-section also decreased by nearly $50 \%$. Monitoring more than 3 times remained constant during the two periods while cases never monitored came down to $0 \%$. Detection of abnormal FHR increased during the COVID period (3.3\%-12.4\%).

\section{Qualitative Findings}

\section{Case load}

In qualitative interviews, some of the respondents mentioned a decrease in case load. Quantitative data show that decrease was in the range of $3 \%$ to almost $50 \%$. Contrarily, one facility in Odisha showed an increase of 5\% during COVID lockdown. A respondent from a $\mathrm{CHC}$ in Jharkhand reported that many patients were refereed to higher centers in the initial phase (of the pandemic), due to uncertainty and lack of operational and technical guidance. However, at the time of the interview, COVID cases were not reported in any center except at the medical college. Although, the program data indicated a decrease in the proportion of C-sections during the lockdown period, was not outside the range of pattern of $\mathrm{C}$ sections reported since the baseline from 2019. The fact that the proportion of detection of abnormal FHR, the number of still births and asphyxia is consistent as in previous months also reveals there is no major difference.

All $(100 \%)$ the study participants reported using handheld Doppler in all labor cases. Four of them used additional devices (Cardiotochograph at the medical college, other Doppler in CHC Mangalore for twin pregnancy, and Stethoscope used by doctor in DH Haridwar) to validate the findings of the intervention Doppler. The device facilitated easy and quick location of FHR, a finding reported by all study participants. However, the strong and wide Doppler beam of the device limits its ability to pick two focal points of heart beat. Therefore, three participants highlighted the challenge of using the handheld device in multiple pregnancies.

\section{Frequency of monitoring}

Quantitative data show that monitoring at least 3 times or more had decreased during COVID time by almost $5 \%$ points, however, cases never monitored also showed a decrease of nearly $4 \%$ points. Frequency of monitoring is not revelatory in itself unless one considers the stage of labor a woman arrives in. Many women come during advanced stage of labor when the delivery is imminent; limiting the number of times they get monitored. Moreover, as some of the respondents mentioned monitoring continuously during COVID times, it might have decreased the number documented, and simultaneously decreased those labor cases which were never monitored $(4.8 \%-0.8 \%)$.

\section{Usefulness of Doppler during COVID times}

Although respondents reported conducting both intermittent as well as continuous monitoring, the latter is currently the practice in many of the facilities - the Medical College and 1 district hospital, $1 \mathrm{SDH}$, and $1 \mathrm{CHC}$. The belt is used under several circumstances: (1) to avoid repeated contact as prevention for COVID, (2) during second stage of labor when frequent monitoring is required, (3) during fetal distress, (4) in primi cases in one facility, (5) when case load is low, and 6) when FHR is normal but meconium is present.

Regarding continuous monitoring during COVID times, this service provider said "Its very convenient to use. We strap on abdomen and we get FHR. We inform attendant to inform us. During our duty we can do other works while woman is getting monitored. Now we are using belts" (LR in charge, SDH Baliguda). The following quote highlights the use of the device in providing a sense of safety to the care providers "Doppler is tied to the woman for continuous monitoring and then nurse does not have to go close to such women again and again" (Staff nurse, RIMS). Use of the belt in continuous monitoring was perhaps dependent on the need for increased cleaning. The nurse from the DH in Jharkhand and CHC, Uttarakhand felt that use of the belt was likely to spread infection. "We use belts but not very often these days since it requires washing and takes time. Hence, we are avoiding continuous monitoring these days" (CHC Mangalore). Although, there was no difference reported between intervention Doppler and other Doppler's in terms of protection during COVID, the use of the belt for continuous monitoring may make a difference as contact between provider and patient is reduced. Many participants believed that using belts may reduce spread of infection during COVID; few others avoided using due to likely spread of infection and the need for cleaning and drying between every patient use which takes time.

\section{IP protocol}

IP protocol was reportedly similar for all women. Enhanced IP practice was revealed in qualitative data. All the pregnant women 
(PW) were given masks to wear. Some PW came wearing their own mask. Personal protective equipment (PPE) was worn by all staff for suspected COVID cases. In all the facilities, precautions were taken. At RIMS, added precaution was taken and the labor room was divided for suspected COVID LR and non-suspected LR. COVID positive women were given surgical mask and cap to wear. Performing frequent hand hygiene was reported by all respondents. Instead of two attendants with the pregnant woman, only one was now allowed as reported by the nurse from $\mathrm{CHC}$ in Jharkhand. She also reported "There is a separate room to receive all women where history is taken. If the pregnant woman is not giving any positive history then only, she comes to the labor room. But so far, even suspected cases have not tested positive."

\section{Cleaning protocol of monitoring device}

Cleaning of the handheld device was done with $70 \%$ ethanol or alcohol spray. Belt was washed with soap water and decontaminated in chlorine solution. Three respondents said they washed the belt in soap water and air dried it. One said, in addition, they used bleaching powder to clean the belt before using. Two respondents said they monitored continuously only in rare cases as belts needed to be cleaned and dried which took time.

\section{Benefits and challenges}

Respondents reported the following benefits of the intervention Doppler:

- Saves time and effort due to continuous monitoring feature

- Portable

- Quick identification of FHR

- Easy to locate FHR, and record available for reference

- Beeping sound and orange color alerts service providers in case there is abnormal FHR

- Indicator for low battery so charging can be done in advance

- Helpful during COVID time as continuous monitoring could be done.

Challenges were faced particularly for twin pregnancy monitoring; in capturing other sounds; and in audibility.

\section{DISCUSSION}

The objective of the study was to assess the experience of using the handheld device during COVID times among service providers, whether FHR monitoring practice was affected by the COVID lockdown period, and whether the IP practices required for the device were helpful in terms of safety of the staff. There was a slight difference in case load during COVID lockdown and previous months. Decrease was not substantial, indirectly signifying the importance of these facilities for childbirth in peoples' perception, so that even during lockdown patients were using the facilities. This does not preclude the importance of looking critically at the decrease, however marginal. A study across primary health facilities found significant disruption of maternal and child health services [16]. The same study also found inadequate supplies of masks, gowns, and hand hygiene amenities. Our study did not report any such lack. Perhaps as most of these were secondary level facilities, they did not experience the same problems. The medical college showed an almost $40 \%$ decrease of cases during the lockdown and almost $50 \%$ decrease in $\mathrm{C}$-sections. In general, it receives people not only from near but also those coming from distant places. Transport was affected during lockdown which prevented people from traveling to the hospital. There was no change in \% cesarean deliveries compared with previous quarter in contrast to some studies which report higher LSCS in COVID positive cases.

In our study, the lockdown period or the suspicion of COVID infection in pregnant women did not affect FHR monitoring significantly. More than 3 times of monitoring for a woman decreased; however, it has to be seen in its context: Perhaps the woman came in advanced stage or the woman was monitored continuously. The practice of continuous monitoring was common for all women in fetal distress and in second stage of labor. It was also seen to be useful as a protection against possible COVID infection as it reduced the need for physical contact. It also had a practical utility in reducing the need for repeated use of the same labor bed, thus possibly reducing the risk of spread of infection. Contradictorily, there were a few other facilities where using the belt for continuous monitoring was not considered useful as it was perceived as high risk for spreading infection. It also necessitated a greater precaution to clean the belt. With increased risk of COVID19 infection among service providers who have to work in close proximity to laboring women [14], the use of continuous monitoring might provide some protection. We need to study this more in depth. Furthermore, feasibility to adopt the practice in smaller facilities with limited resources (limited number of Doppler's and staff) must be examined before we can reach any conclusion.

As the interviews were conducted in late May and early June, COVID cases were not too many due to the total lockdown in the country. The major limitation of the entire study was that we could not seek information about how the handheld device is able to help providers who are directly dealing with COVID positive cases except at the medical college. The data, however, inform about the extra precautions being followed in receiving and monitoring pregnant women in the facility. The other limitation was that we could not assess the risk perception and gauge fear of service providers delivering COVID positive cases. For suspected cases, they did mention that they either referred to a higher facility or wore full PPE kit: Mask, gloves, and goggles, which is in compliance with guidelines. In addition, since our sample size was small and validating these findings was not possible as interviews were conducted remotely.

\section{CONCLUSION}

The use of the handheld Doppler was found to be consistent in COVID times as in other periods. With the use of belt for continuous monitoring, it might confer an added benefit as a protective device. However, since the assessment was done at the beginning of the surge in COVID cases, we cannot conclusively 
deduce this as the assessment for the current phase. IP protocol during the lockdown period was found to be sound indicating the timely issuance of guidelines and remote trainings and reinforcing support facilitated by Vriddhi project. Frequency of FHR monitoring and detection of abnormal FHR was not affected, suggests the sound knowledge of health-care providers on the importance of FHR monitoring regardless of the situation.

\section{AUTHOR CONTRIBUTION}

DB conceptualized the intervention, oversaw data collection, and contributed to writing of the article; RA acquired data, made significant contribution to analysis and writing of article; SGD, NB and RKP implemented the intervention, conducted supervision, monitored data quality and reviewed the paper; AK managed and analyzed the data, ES wrote the first draft, analyzed qualitative data and revised the article, HK conceptualized the study and critically reviewed the article.

\section{ACKNOWLEDGMENT}

We are grateful to the study participants for providing us the time for interviews. We are grateful to our state implementation teams. We own gratitude to the State Governments of Jharkhand, Odisha and Uttarakhnad.

\section{REFERENCES}

1. Kassebaum NJ, Bertozzi-Villa A, Coggeshall MS, Shackelford KA, Steiner C, Heuton KR, et al. Global, regional, and national levels and causes of maternal mortality during 1990-2013: A systematic analysis for the Global Burden of Disease Study 2013. Lancet 2014;384:980-1004.

2. Say L, Chou D, Gemmill A, Tunçalp Ö, Moller AB, Daniels J, et al. Global causes of maternal death: A WHO systematic analysis. Lancet Glob Health 2014;2:e323-33.

3. Lawn JE, Blencowe H, Waiswa P, Amouzou A, Mathers C, Hogan D, et al. Stillbirths: Rates, risk factors, and acceleration towards 2030. Lancet 2016;387:587-603.

4. World Health Organization. India has Achieved Groundbreaking Success in Reducing Maternal Mortality. Geneva: World Health Organization; 2018 Available from: http://www.searo.who.int/mediacentre/features/2018/indiagroundbreaking-sucess-reducing-maternal-mortality-rate/en. [Last accessed on 2019 May 12].
5. Million Death Study Collaborators. Changes in cause-specific neonatal and 1-59-month child mortality in India from 2000 to 2015: A nationally representative survey. Lancet 2017;390:1972-80.

6. Kc A, Wrammert J, Clark RB, Ewald U, Målqvist M. Inadequate fetal heart rate monitoring and poor use of partogram associated with intrapartum stillbirth: A case-referent study in Nepal. BMC Pregnancy Childbirth 2016;16:233.

7. Yisma E, Dessalegn B, Astatkie A, Fesseha N. Completion of the modified World Health Organization (WHO) partograph during labour in public health institutions of Addis Ababa, Ethiopia. Reprod Health 2013;10:23.

8. Qiao J. What are the risks of COVID-19 infection in pregnant women? Lancet 2020;395:760-2.

9. Chen D, Yang H, Cao Y, Cheng W, Duan T, Fan C, et al. Expert consensus for managing pregnant women and neonates born to mothers with suspected or confirmed novel coronavirus (COVID-19) infection. Int J Gynaecol Obstet 2020;149:130-6.

10. Chen H, Guo J, Wang C, Luo F, Yu X, Zhang W, et al. Clinical characteristics and intrauterine vertical transmission potential of COVID-19 infection in nine pregnant women: A retrospective review of medical records. Lancet 2020;395:809-15.

11. Jiao J. Under the epidemic situation of COVID-19, should special attention to pregnant women be given? J Med Virol 2020;92:1371-2.

12. Mullins E, Evans D, Viner RM, O'Brien P, Morris E. Coronavirus in pregnancy and delivery: Rapid review. Ultrasound Obstet Gynecol 2020;55:586-92.

13. Panahi L, Amiri M, Pouy S. Risks of novel coronavirus disease (COVID-19) in pregnancy; a narrative review. Arch Acad Emerg Med 2020;8:e34.

14. Palatnik A, McIntosh JJ. Protecting labor and delivery personnel from COVID-19 during the second stage of labor. Am J Perinatol 2020;37:854-6.

15. Stephens AJ, Barton JR, Bentum NA, Blackwell SC, Sibai BM. General guidelines in the management of an obstetrical patient on the labor and delivery unit during the COVID-19 pandemic. Am J Perinatol 2020;37:829-36.

16. Garg S, Basu S, Rustagi R, Borle A. Primary health care facility preparedness for outpatient service provision during the COVID-19 pandemic in India: Cross-sectional study. JMIR Public Health Surveill 2020;6:e19927.

Funding: This work was made possible by the support of the American people through the United States Agency for International Development and its VRIDDHI (scaling up RMNCH + A Interventions) Project, implemented by IPE Global Ltd. under the terms of Cooperative Agreement Number AID $386 \mathrm{~A}$ 1400001 . The contents of this paper represent the views of the authors and do not reflect the views of the US Government; Conflict of Interest: None Stated.

How to cite this article: Bajpayee D, Agrawal R, Dastidar SG, Bisht N, Panda RK, Kumar A, Sarin E, Kumar H. Experience with the use of handheld Doppler for fetal heart rate monitoring during coronavirus disease-19 pandemic. East J Med Sci. 2020;5(4):74-78.

Doi: 10.32677/EJMS.2020.v05.i04.002 